\title{
PROMOTE patency-does it have the answers?
}

\author{
Pradeep Narayan ${ }^{1}$ (iD
}

Received: 15 August 2019 /Revised: 5 September 2019 / Accepted: 22 September 2019 / Published online: 15 November 2019

(C) Indian Association of Cardiovascular-Thoracic Surgeons 2019

The "prospective randomized comparison of off-pump and on-pump multivessel coronary artery bypass surgery (PROMOTE patency) trial" is the first multicenter randomized controlled study that compared off-pump with on-pump coronary artery revascularization in the Indian population [1]. For this, the investigators of this study have to be congratulated. The study was meticulously planned, well conducted, and very extensively analyzed.

The primary outcome of the study was assessment of patency of grafts at 3 months which adds further credibility to the trial as the authors did not limit themselves to the clinical outcomes alone. The study design was based upon another randomized controlled trial (RCT) by Khan et al. which also studied angiographic patency of grafts at 3 months [2]. Interestingly, the angiographic follow-up at 3 months was quite similar in both the studies, $-76 \%$ in the PROMOTE patency and $79 \%$ in the study by Khan et al. However, unlike the study by Khan et al. which showed a much inferior patency in the off-pump group ( $88 \%$ vs. $98 \% ; p=0.002)$, the PROMOTE patency group has demonstrated a similar patency in both arms $(87.1 \%$ vs. $87.6 \%, p=0.8)$. This could be due to the fact that while the 2 surgeons in the study by Khan et al. had previously carried out only $13 \%$ of the coronary revascularization using the off-pump technique, in the PROMOTE patency group, the surgeons were required to have carried out at least $50 \%$ of their coronary revascularizations using the off-pump technique in the preceding 3 years.

Experience of surgeons is an important issue and appears relevant to the PROMOTE patency trial as well. In the past, the Randomized On/Off Bypass (ROOBY) trial had been criticized for keeping the entry criteria for participating in the trial at 20 off-pump cases [3]. The Arterial Revascularization Trial

Pradeep Narayan

pradeepdoc@gmail.com

1 Department of Cardiac Surgery, NH Rabindranath Tagore International Institute of Cardiac Sciences, Kolkata 700099, India
(ART) stipulated that "only surgeons with experience of 50 bilateral internal mammary artery (BIMA) operations were able to undertake BIMA procedures in the trial," and despite this, significant variability was observed among the outcomes of participating surgeons [4]. The PROMOTE patency trial laid out much stricter criteria and stipulated that only surgeons who had "performed more than 500 coronary artery bypass grafting $(\mathrm{CABG})$ procedures (250 procedures of each technique) during the 3 years prior to the trial" would participate in the trial [1]. However, at this point, it has to be said that generally, coronary surgeons are either predominantly "offpump surgeons" or "on-pump surgeons." It is unusual for surgeons to carry out coronary revascularization using both techniques routinely. So it is difficult to understand how it was ensured that this criterion of " 250 procedures in each technique" was met by all participating surgeons. Did the researchers adhere to this requirement or was the overall experience and familiarity with both techniques taken as a reasonable, and in my view completely acceptable, compromise? Also, do the researchers feel that adopting an expertise-based model, in any given technique, as laid out in the CABG Off or On Pump Revascularization Study (CORONARY) would have been a better option for judging expertise? [5].

Another very important issue with regard to the PROMOTE patency trial is using two different modalities to assess graft patency and is perhaps the most important confounder of the trial. Graft patency was assessed at 3 months by either 128 slice multidetector-computed tomography (MDCT) or conventional catheter coronary angiography $(\mathrm{CAG})$. There is no denying that 128 slice multidetector-computed tomography (MDCT) is comparable with conventional angiography in terms of sensitivity and specificity. However, both the systems while being completely valid individually use a different system of evaluation, and to the best of my knowledge, there is no system of amalgamating these two evaluation systems. It is very akin to classification of aortic dissections using DeBakey and Stanford classification. While both the systems are completely valid, a type II aortic dissection (limited entirely to ascending aorta) cannot be considered the same as type A 
aortic dissection (ascending aorta \pm distal aorta) in the Stanford system. So how did the authors ensure that the graft evaluation performed by the MDCT was made comparable with FitzGibbon's grading of grafts (A, B, O)? Also, could the authors have chosen to analyze the findings of these two modalities separately?

The argument provided by the authors in support of using both modalities is that "graft evaluation was not uniform due to non-availability of MDCT facility at some centers where the graft evaluation was done by catheter CAG." However, this is inaccurate as the study was designed to evaluate patency at 3 months in all participants using conventional angiography as mentioned in the Clinical Trials Registry of India (CTRI/2017/10/010030) document enclosed with the manuscript. So why did the authors deviate from their original protocol? The reason this question has to be raised is not to highlight the protocol deviation but for gaining insight into the issue that will allow future researchers to surmount any obstacles the PROMOTE patency trialists may have encountered.

One of the exclusion criteria that the authors mention in the protocol submission to CTRI is "contra-indications to offpump CABG or on-pump CABG (calcified aorta, intramuscular left anterior descending (LAD), calcified coronaries, small target vessels, resection of ventricular aneurysm)." As calcified aorta, intramuscular LAD, and small target vessels can often only be identified intra-operatively (and thus postrandomization), did the authors remove this exclusion criteria in the actual trial? If they did apply this in the trial, were there any patients excluded from the trial using this criterion?

While there are no issues with the power of the study, the authors calculated the required sample size as 310 patients (155 patients in each arm), and to compensate for eventual dropouts of $10 \%$, they kept an enrolment target of 350 patients (175 patients in each arm). The sample size mentioned in the CTRI was even higher at 400 . The study finally enrolled only 320 patients which was still above the required sample size and had sufficient power to support the conclusions drawn by the authors. However, what was the reason for truncating the recruitment of patients at 320 which was below their initial target of 350 ?

The final issue relates to generalizability of the trial findings. The patient population in the trial setting was extremely low risk (EuroSCORE II being 0.98 in both arms). Do the authors feel that findings of this trial can be extrapolated to the population at large?

A perfect trial is perhaps a utopian concept, and none of the questions raised should distract the readers from the fact that the PROMOTE patency is so far the only multicentric trial coming out of India which has examined the non-inferiority of off-pump technique in such a robust fashion. It is also the first time that data from the Indian populace has been made available on such a scale in coronary literature. The findings of the trial are reassuring to a large number of surgeons in the country who use the off-pump technique for coronary revascularization, and the authors once again have to be congratulated for their endeavors.

Compliance with ethical standards The invited editorial does not mention any patient or patient details, and hence, formal ethical approval is not required.

Conflict of interest The authors declare that they have no conflict of interest.

\section{References}

1. Sajja LR, Sarkar K, Mannam G, et al. Graft patency at 3 months after off- and on-pump coronary bypass surgery: a randomized trial. Indian J Thorac Cardiovasc Surg. https://doi.org/10.1007/s12055019-00869-0.

2. Khan NE, De Souza A, Mister R, et al. A randomized comparison of off-pump and on-pump multivessel coronaryartery bypass surgery. $\mathrm{N}$ Engl J Med. 2004;350:21-8.

3. Shroyer AL, Grover FL, Hattler B, et al. On-pump versus off-pump coronary-artery bypass surgery. N Engl J Med. 2009;361:1827-37.

4. Taggart DP, Benedetto U, Gerry S, et al. Bilateral versus single internal-thoracic-artery grafts at 10 years. N Engl J Med. 2019;380: 437-46.

5. Lamy A, Devereaux PJ, Prabhakaran D, et al. Off-pump or on-pump coronary-artery bypass grafting at 30 days. N Engl J Med. 2012;366: 1489-1497.

Publisher's note Springer Nature remains neutral with regard to jurisdictional claims in published maps and institutional affiliations. 